\title{
Cytoreductive Surgery and Hyperthermic Intraperitoneal Chemotherapy for Recurrent Epithelial Ovarian Cancer, the First Egyptian Experience
}

\author{
Gareer WY ${ }^{1}$, Gareer $\mathrm{HWY}^{1}$, Hussein Alaadin ${ }^{1 *}$, Mansour $\mathbf{0}^{2}$, Alghazali $\mathrm{H}^{3}$ and Saber $\mathrm{A}^{4}$ \\ ${ }^{1}$ Surgical Oncology, National Cancer Institute, Cairo University, Egypt \\ ${ }^{2}$ Medical Oncology Departments, National Cancer Institute, Cairo University, Egypt \\ ${ }^{3}$ Medical Oncology Department, Ain-Shams University, Egypt \\ ${ }^{4}$ Medical Oncology Department, Menia University, Egypt
}

Submission:June 27, 2017; Published: July 14, 2017

*Corresponding author: Hussein Alaadin, Surgical Oncology, National Cancer Institute, Cairo University, Egypt, Email: alaadin.osman@yahoo.com

\begin{abstract}
Background: For 3 decades cytoreductive surgery (CRS) and hyperthermic intraperitoneal chemotherapy (HIPEC) has been introduced as an effective technique in treating peritoneal carcinomatosis (PC) from different primaries with promising results in selected patients .The Aim of this study is to evaluate the early and late outcomes of CRS and HIPEC in management of recurrent epithelial ovarian cancer (REOC) with PC as the first Egyptian experience.
\end{abstract}

Methods: In the period between September 2009 and December 2013 we treated 42 patients with PC from REOC with CRS and HIPEC using the closed abdominal technique. The perioperative complications, toxicity grade, overall survival (OS), disease free survival (DFS), mortality rates, and effects of peritoneal carcinomatosis index (PCI), completeness of cytoreduction, patient age and tumor type and grade on survival had been reported and analyzed.

Results: The mean age was $51.2 \pm 7.9$ (range $34-64$ years). PCI was $<16$ in $47.7 \%$; $16-20$ in $45.2 \%$ and $>20$ in $7.1 \%$ ). Postoperative mortality rate was $2.38 \%$. The mean disease free survival (DFS) was $11.9 \pm 9.75$ (median 9.0; range 0-51 months), while the cumulative overall survival (OS) was $73.6 \%$ at 18 months, and $55.5 \%$ at 2 years.PCI found to be a significant risk factor when we compared PCI $<16$ with PCI $>16(p=0.001$. Completeness of cytoreduction was associated with higher OS. Age $>60$ years was associated with a significantly lower OS $(p=0.003)$. Serous papillary tumors were associated with better survival. Low tumor grade was associated with longer OS survival with no significant difference (0.628).

Conclusion: CRS and HIPEC along with the extent of the disease and the extent of cytoreduction play a crucial role in the survival of patients with REOC, and as a technique it is feasible with acceptable morbidity, mortality (2.38\%) and toxicity when good patients' selection and systematic intra operative assessment are strictly applied.

Keywords: Recurrent epithelial Ovarian Cancer; Cytoreductive surgery; Intraperitoneal hyperthermic chemotherapy

Abbreviations: CRS: Cytoreductive Surgery; HIPEC: Hyperthermic Intraperitoneal Chemotherapy; PC: Peritoneal Carcinomatosis; REOC: Recurrent Epithelial Ovarian Cancer; OS: overall survival; DFS: Disease Free Survival; PCI: Peritoneal Carcinomatosis Index; EOC: Epithelial ovarian cancer

\section{Introduction}

Epithelial ovarian cancer (EOC) is The most common cause of primary ovarian malignancy accounting for $95 \%$ of ovarian neoplasms, with a lifetime risk of 1 in every 70 women [1,2], making it the sixth commonest malignancy in women worldwide,

and it is one of the leading causes of cancer related deaths in women with over 100,000 deaths due to the disease each year worldwide $[3,4]$. It has an indolent clinical course leading to most cases being diagnosed in an advanced stages (FIGO stage III,IV) with about $75 \%$ of cases having peritoneal deposits at 
time of diagnosis $[5,6]$, the 5 year survival for such patients is $>25 \%$ with the standard therapy being maximal cytoreductive surgery followed by platinum-based chemotherapy [7-9] which despite having $70-80 \%$ response rate still about $75 \%$ of these patients will have recurrent disease mostly confined to the peritoneal cavity with a median disease free survival 18-28 month only $[10,11]$ ultimately suffering from bowel obstruction and death and thus the need for better loco regional control for such advanced stages [12,13].

Another approach which was originally described by Sugar baker in 1980's combines surgery with HIPEC with surgery aiming at complete removal of tumor burden through peritonectomy and visceral resection to remove all macroscopically identified tumor $[14,15]$ and during the last decade reports with good results are being published from many centers $[7,16]$ and survival improvement is mainly dependent on completeness of cytoreduction thus supporting the key role of radical surgery in REOC treatment [17].

\section{Patients and Methods}

Between September 2009 and December 2013, our team treated 42 women who experienced recurrence after initial treatment with cytoreductive surgery and systemic chemotherapy. All cases were submitted to complete labs (blood count, blood chemistry tests, urine analysis and Ca125 serum levels), Pelvi-abdominal CT scan and PET-CT scan to exclude extra-abdominal disease and assess disease extension and the chances of optimal cytoreduction. The PCI was calculated by summing the tumor volume in each of the 13 abdomino-pelvic regions defined by Sugar baker [18]. Patients' inclusion criteria included: women aged between 34 and 68 years with recurrent ovarian cancer; Eastern Cooperative Group Performance Status (ECOG PS) $\leq 2$; no evidence of disease outside the abdomen. No extensive bowel involvement, absence of non-resectable liver metastasis, and with normal cardiac, hepatic, renal, respiratory and bone marrow functions. The exclusion criteria were as follows: ECOG PS <2; evidence of extra-abdominal metastatic disease, presence of non-respectable liver metastasis, extensive bowel obstruction or peri-portal involvement or any intraabdominal involvement that will not result in CC-0 or CC-1 cytoreduction. The protocol was approved by the Ethical Committee of the hospital, and an informed consent was signed by all patients.

\section{Surgical Technique}

All patients were operated on by the same surgical team and under general anesthesia we started our procedure with proper exploration to properly calculate the PCI and access operability. Peritonectomy procedure was tailored according to the extent of disease. Affected organs were resected in bloc with affected pelvic peritoneum. Surgical procedures done are summarized in (Table 1). Retroperitoneal and pelvic lymphadenectomy were performed when affected. The completeness of cytoreduction was assessed according to Sugar baker classification CC-0; no residual disease; CC-1; minimal residual disease $<2.5 \mathrm{~mm}$; CC-2, residual disease of $2.5 \mathrm{~mm}-2.5 \mathrm{~cm}$; and CC-3, disease $>2.5 \mathrm{~cm}$. At the end of CRS we started the HIPEC using the closed technique using Cisplatin (CDDP $50 \mathrm{mg} / \mathrm{m} 2$ ) +Doxorubicin (15mg/m2) or Cisplatin-Mitomycin C $(25 \mathrm{mg}-3.3 \mathrm{mg} / \mathrm{m} 2 / \mathrm{L})$. The perfusion continued for 90 minutes at an average temperature of $42-43^{\circ} \mathrm{C}$. At the end of the procedure, restoration of bowel continuity for any resected bowel is established.

\section{Follow-Up}

The patients were followed up every 3 months during the first 2 years after surgery and every 6 months later with physical examination, hematological-biochemical examinations, tumor markers (CEA, CA-125), and pelvi-abdominal CT scan and PET scan for cases with elevated Ca-125 and negative CT scan. The recurrences and the sites of recurrence were recorded (Table 1).

Table 1: Surgical procedures.

\begin{tabular}{|c|c|c|}
\hline Peritonectomy \& Other procedures & $\begin{array}{l}\text { Number } \\
\text { of cases }\end{array}$ & percentage \\
\hline Rt. sub diaphragmatic & 38 & \\
\hline Lt. sub diaphragmatic & 31 & \\
\hline Completion of greater omentectomy & 37 & \\
\hline Lesser omentectomy & 1228.6 & \\
\hline Pelvic peritonectomy & 36 & 85.7 \\
\hline $\begin{array}{l}\text { Pelvic Peritonectomy+ partial } \\
\text { cystectomy }\end{array}$ & 2 & 4.76 \\
\hline $\begin{array}{l}\text { Pelvic Peritonectomy +Anterior } \\
\text { resection of the rectum }\end{array}$ & 49.52 & \\
\hline Right parietal & 32 & \\
\hline Left parietal & 29 & \\
\hline Cholecystectomy & 25 & 59.5 \\
\hline Splenectomy & 19 & 45.2 \\
\hline Small bowel resection & 9 & 21.4 \\
\hline Right colectomy & 8 & 19 \\
\hline Subtotal colectomy & 5 & 11.9 \\
\hline $\begin{array}{c}\text { Pelvic, Para-aortic lymph node } \\
\text { resection }\end{array}$ & 12 & 28.6 \\
\hline Partial gastrectomy & 2 & 4.76 \\
\hline
\end{tabular}

\section{Statistical Analysis}

All data were collected prospectively. Descriptive statistics were generated for all measures, Including means, ranges, and standard deviations for continuous measures and frequencies and proportions for categorical data. OS was calculated from the date of surgery to the last known date of follow-up or the date of death. Estimates of survival were calculated by using the Kaplan-Meier (product-limit) method; analysis with Cox proportional hazards was performed on all pertinent clinicopathologic variables to determine each variable's association with survival. Group comparisons of OS were performed 


\section{Cancer therapy \& Oncology International Journal}

by using the approximate v2 statistic for the log-rank test. Additionally, the Cox proportional-hazards regression model was used in a stepwise fashion to perform a multivariate analysis of clinicopathologic factors to determine an overall model of independent predictors of OS. Statistical significance was defined as a $\mathrm{P}$ value $<.05$.

\section{Results}

In 42 patients the mean age was $51.2 \pm 7.9$ (range 34-64 years), PCI was $<16$ in $47.7 \% ; 16-20$ in $45.2 \%$ and $>20$ in $7.1 \%$. The mean operative time was $366 \pm 69 \mathrm{~min}$ (range $230-510 \mathrm{~min}$ ); with a blood loss mean of $883 \pm 389$ cc, (range 280-2100 cc.). Completeness of cytoreduction was: CC- 0 in $66.7 \%$ and CC-1 in $33.3 \%$ of patients. The mean hospital stay was $12 \pm 6$ days (range 6-20 days) among which the mean intensive care unit (ICU) stay was $5 \pm 2$ days (range 1-20 days).

Postoperative morbidity (according to The National Cancer
Institute's Common Terminology Criteria for Adverse Events (CTACE) version 4.03 updated in June, 2010) and mortality are presented in (Table 2). The mean disease free survival (DFS) was 11.9 \pm 9.75 (median 9.0; range 0-51 months), while the cumulative overall survival (OS) was $73.6 \%$ at 18 months, and $55.5 \%$ at 2 years (Figure 1). Patient age had an effect on OS with Age $>60$ years was associated with a significantly lower OS ( $p=0.003)$, PCI was found to be a significant risk factor when we compared PCI $<16$ with PCI $>16(\mathrm{p}=0.001)$ (Figure 2), Completeness of cytoreduction (whatever the PCI) was associated with apparently higher OS proportion, but there was no significant difference between CC-0 and CC-1 ( $p=0.125)$ (Figure 3), tumor grade affect OS with no statistical difference $(p=0.225)$. The Different risk factors in all patients including age, type of pathology, tumor grade, PCI and completeness of cytoreduction and their relation to cumulative survival are presented in (Table 3).

Table 2: Postoperative morbidity and mortality.

\begin{tabular}{|c|c|c|}
\hline Complication & Number & Percentage \\
\hline Grade I & 21.42 & 21.42 \\
\hline Prolonged ileus & & \\
\hline Grade II & 9 & \\
\hline Nausea & 49.52 & 14.2 \\
\hline Urinary tract infection & 5 & 9.52 \\
\hline Pleural effusion & 6 & 7.14 \\
\hline Wound infection & 4 & 4.76 \\
\hline Neutropenia grade II & 3 & 4.76 \\
\hline Pneumonitis & 2 & \\
\hline Renal impairment & 2 & \\
\hline Enterocutaneous fistulas & & \\
\hline Grade III (Reintervention) & 2 & \\
\hline Bleeding & 1 & 2.38 \\
\hline GI fistulas & 1 & 2.38 \\
\hline Grade IV & 2 & 4.76 \\
\hline Anastomotic leak \& peritonitis & 12.38 & \\
\hline Mortality & & \\
\hline
\end{tabular}

Table 3: Risk factors and their relation to cumulative survival.

\begin{tabular}{|c|c|c|c|}
\hline Factor & $\mathbf{n}$ & Cumulative Survival (\%) & p value \\
\hline Age & & & $32.5 \%$ \\
\hline$>60$ yrs. & 24 & $84.6 \%$ & \\
\hline$\leq 60$ yrs. & 18 & & \\
\hline Pathology & & $76.2 \%$ & \\
\hline Serous & 22 & $35.4 \%$ & \\
\hline Non-serous (Mucinous) & 16 & & \\
\hline Undifferentiated & 4 & & \\
\hline Grade & & & \\
\hline
\end{tabular}


Cancer therapy \& Oncology International Journal

\begin{tabular}{|c|c|c|c|}
\hline 1 & 4 & $64.2 \%$ & 0.225 \\
\hline 2 & 22 & $50.0 \%$ & $41.5 \%$ \\
\hline 3 & 16 & & 0.125 \\
\hline Completeness of cytoreduction & & $74.7 \%$ & \\
\hline CC0 & 28 & $35.7 \%$ & \\
\hline CC1 & 14 & & 0.001 \\
\hline PCI & 27 & $86.1 \%$ & $15.8 \%$ \\
\hline
\end{tabular}

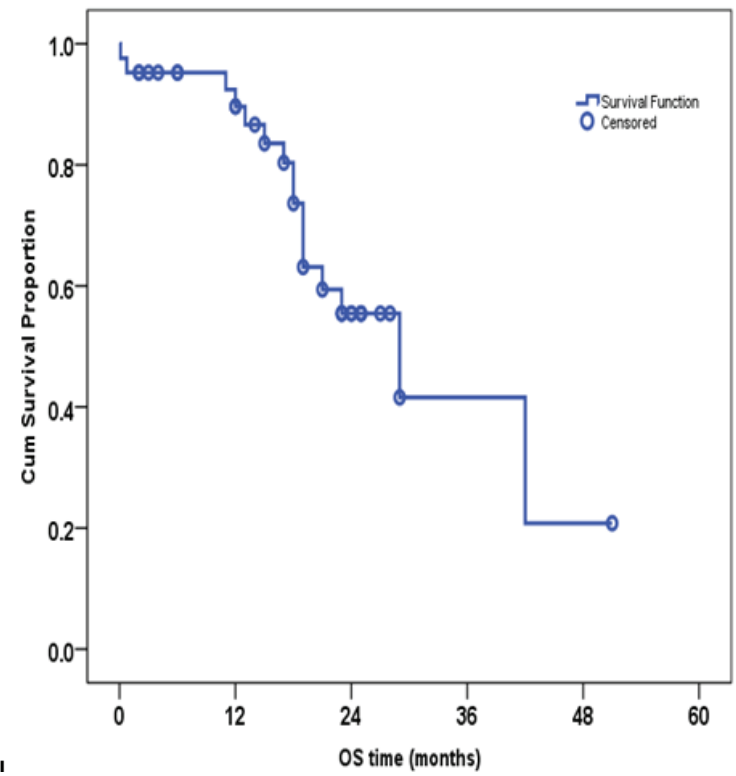

Figure 1: cumulative overall survival (OS).

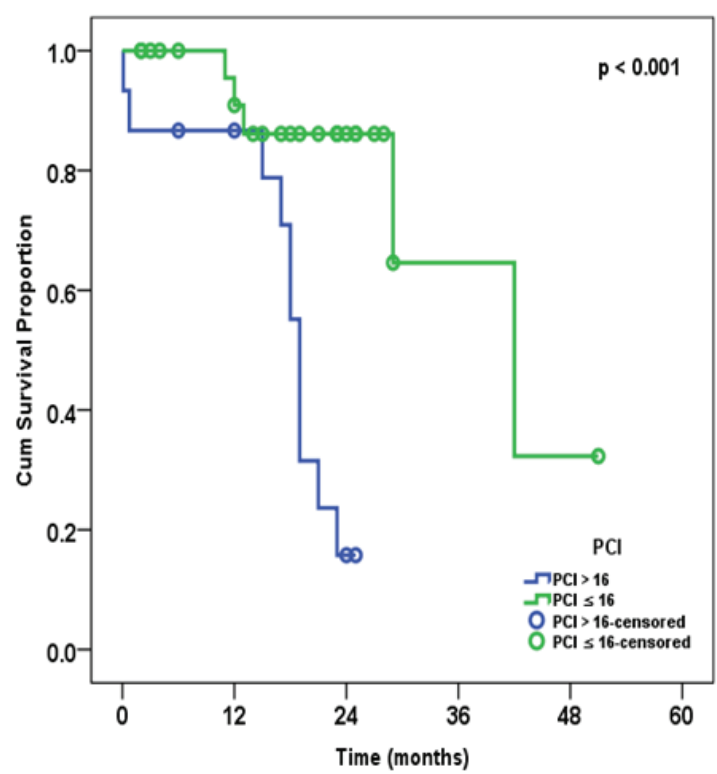

Figure 2: The relation between $\mathrm{PCl}$ and cumulative survival.

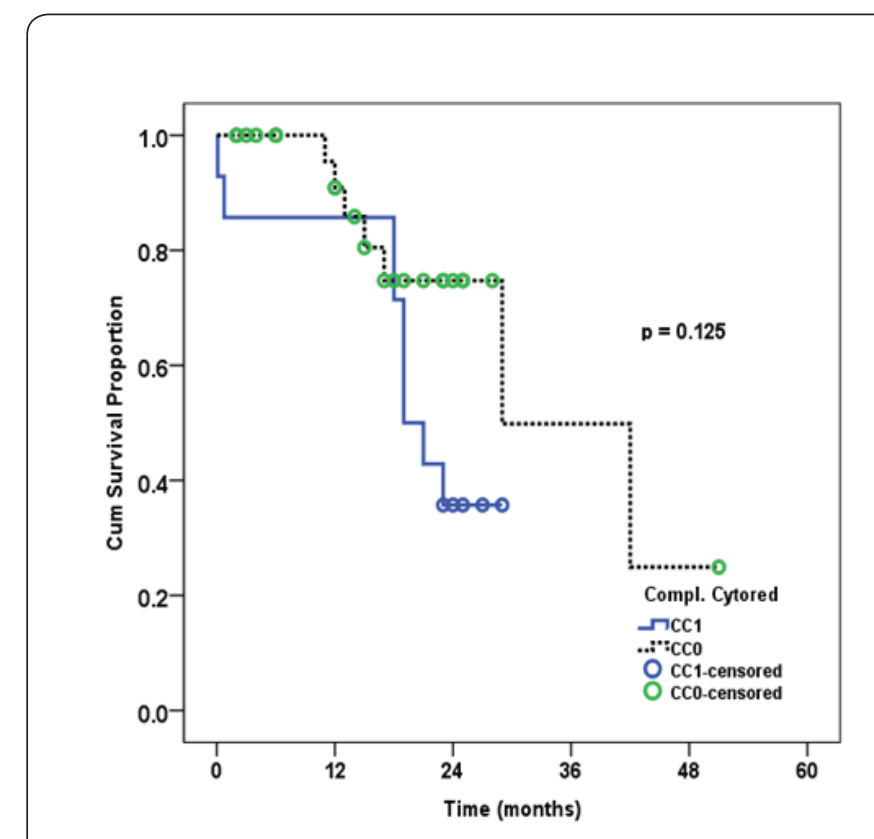

Figure 3: Cumulative survival in relation to Completeness of cytoreduction.

\section{Discussion}

EOC has an indolent course and more than $60 \%$ of cases are diagnosed in advanced stages and also it tends characteristically to disseminate intraperitoneally. Most of these cases experience recurrence in first 3 years after diagnosis and treatment thus the need for better local control [19]. CRS and HIPEC has been implemented as a treatment for EOC with peritoneal deposits and have shown to be efficient when applied either as (interval HIPEC) meaning after neoadjuvant chemotherapy without previous surgery or as (consolidation HIPEC) after initial CRS and a full course of adjuvant chemotherapy in patients with a clinically complete response [20].

The importance of HIPEC in treatment of REOC was proved in a recent randomized trial by J Spiliotis et al. [19] where the patients were classified into 2 groups, the first group was treated with CRS and HIPEC followed by chemotherapy and the second group were only treated by CRS and chemotherapy. They reported a significant difference in survival, as they found CCO in HIPEC limb survival 30.9 month compared to 16.1 month in CC0 without HIPEC thus highlighting the beneficial effect of HIPEC 


\section{Cancer therapy \& Oncology International Journal}

over CRS only [19]. Another work by Pedro Antonio et al. also measured disease free survival after 1 and 2 years and it was 81 $\%$ and $67 \%$ with HIPEC and only $66 \%$ and $33 \%$ in CRS without HIPEC [21], this confirms the importance of HIPEC after CRS and in our work comparative results were found as we had an overall survival of $73.6 \%$ at 18 month and $55.5 \%$ at 2 years with a mean DFS $11.9 \pm 9.75$ month.

The extent of cytoreduction is one of the most important prognostic factors, and it improves greatly oS whatever the disease stage if complete cytoreduction (CC-0 or CC- 1 ) is followed by HIPEC [22]. In our study the completeness of cytoreduction had a great effect on the cumulative survival which was $74.7 \%$ and $35.7 \%$ with CC0 and CC1 respectively, this was also supported by various studies as Ingmar konigsvainer et al. [23] who stated in his study which included 90 patients that those who had $\mathrm{CC} 0-1$ had better overall survival than those who had CC2-3 cytoreduction, also Rene Warschkow et al. [24] published similar results and among their 52 patients those who had CC0 their survival was $71 \%$ at 2 years and $63 \%$ at 5 years and these numbers decreased with CC1-2 patients(39 patient) to $36.7 \%$ and $17 \%$, and further decreased with CC2 patients (20 patient) to $17.5 \%$ and $0 \%$ at 2 years and 5 years respectively [24], also J Spiliotis et al. [19] results confirmed the importance of complete cytoreduction with CC0 , CC1, CC2 having survival of $30.9,23.9$ and 12.1 month respectively.

Mulier S et al. [25] published their results on recurrent EOC patient series, and their median OS and median disease-free survival after CRS and HIPEC followed by adjuvant chemotherapy were 15-57 months and 3-48 months, respectively, while 5-year OS and 5-year disease-free survival were 18-57 \% and 0-12.5 $\%$ respectively When a complete cytoreduction was achieved, median OS was 97.4 months and 5-year OS was 63-67\%. Completeness of cytoreduction was confirmed as the strongest predictor of survival in many other series $[20,26]$.

PCI is an important independent factor in determing survival and in our work we compared PCI $>16$ and PCI $<16$ with the overall survival being $86.1 \%$ and $15.8 \%$ respectively, a result that was supported by J Spiliotis et al. [19] whose results showed that cases with $\mathrm{PCI}>15$ had better survival of 30.4 month versus 21.5 month in cases with $\mathrm{PCI}<15$, even in their Non HIPEC limb of the study it was 15.4 month versus 9.2 month respectively for same PCI groups [19], in another study they stated that PCI does not affect survival so long as complete cytoreduction could be achieved, in another words the effect of high PCI is a result of higher incidence of inability to reach CC0 [23].

A recent review stated that the morbidity and mortality associated with HIPEC which was the main concern, has declined over the years and is now comparable to that of CRS alone, and they reviewed various studies with acceptable morbidity and mortality rates, with the Major complications being anastomotic leakage, bowel perforation, and intraperitoneal hemorrhage and wound dehiscence [27]. In our study the rate of complications was comparable with prolonged ileus and nausea being the most common and happened in $21.42 \%$ of patients, wound infection in $14.2 \%$, leakage with peritonitis in only 2 patients $(4.76 \%)$ and mortality in only 1 of our 42 patients (2.38\%).

\section{Conclusion}

Cytoreductive surgery and HIPEC for ovarian tumors with peritoneal metastasis is feasible with acceptable morbidity and mortality when good patients' selection and systematic intra operative assessment are strictly applied. And it offers a significant survival benefit over cytoreductive surgery alone especially when complete cytoreduction CCO could be achieved.

\section{Conflict of Interest}

The authors declare that there is no conflict of interest regarding the publication of this paper.

\section{References}

1. Surveillance (2014) Epidemiology, and End Results (SEER). National Cancer Institute, USA.

2. Siegel R, Naishadham D, Jemal A (2013) Cancer statistics. CA Cancer J Clin 63(1): 11-30.

3. Parkin DM, Bray F, Ferlay J (2005) Global cancer statistics. CA Cancer J Clin 55(2): 74-108.

4. Bristow R (2007) Primary Cytoreductive Surgery for Advanced Stage Ovarian Cancer: indications for Radical Resection. Cancerología 1: 3136.

5. Slomovitz B, Soliman P, Wolf J (2006) Gynecologic Cancers. The MD Anderson surgical oncology handbook. (4th edn), Lippincott Williams and Wilkins, Philadelphia, USA.

6. Sehouli J, Senyuva F, Fotopoulou C(1999) Neumann U Intra-abdominal tumor dissemination pattern and surgical outcome in 214 patients with primary ovarian cancer J Surg Oncol 99(7): 424-427.

7. Di GA, Naticchioni E, Biacchi D (2008) Cytoreductive surgery (peritonectomy procedures)combined with hyperthermic intraperitoneal chemotherapy (HIPEC) in the treatment of diffuse peritoneal carcinomatosis from ovarian cancer. Cancer 113(2): 31525.

8. Bookman MA, McGuire WP, Kilpatrick D (1996) Carboplatin and paclitaxel in ovariancarcinoma: a phase I study of the Gynecologic Oncology Group. J Clin Oncol 14(6): 1895-902.

9. Deraco M, Rossi CR, Pennacchioli E (2001) Cytoreductive surgery followed by intraperitoneal hyperthermic perfusion in the treatment of recurrent epithelial ovarian cancer: a phase II clinical study. Tumori 87: $120-126$.

10. BakrinN, ClasseJM, PomelC, GouyS (2014) Hyperthermic intraperitoneal chemotherapy (HIPEC) in ovarian cancer. J ViscSurg 151(3): 347-353.

11. Bae JH, Lee JM, Ryu KS (2007) Treatment of ovarian cancer with paclitaxel- or carboplatin-based intraperitoneal hyperthermic chemotherapy during secondary surgery. Gynecol Oncol 106(1): 193200 .

12. Armstrong DK (2002) Relapsed ovarian cancer: challenges and 


\section{Cancer therapy \& Oncology International Journal}

management strategies for a chronic disease. Oncologist 7: 20-28.

13. Gori J, Castano R, Toziano (2005) Intraperitoneal hyperthermic chemotherapy in ovarian cancer. Int J Gynecol Cancer 15(2): 233-239.

14. Sugarbaker PH (1996) Peritonectomy procedures. Cancer Treat Res 221(1): 235-253.

15. Mohamed F, Cecil T, Moran B, Sugarbaker P (2011) A new standard of care for the management of peritoneal surface malignancy. Curr Oncol 18(2): 84-96.

16. Pomel C, Ferron G, Lorimier G (2010) Hyperthermic intra-peritoneal chemotherapy using oxaliplatin as consolidation therapy for advanced epithelial ovarian carcinoma. Results of a phase II prospective multicenter trial. CHIPOVAC study. Eur J Surg Oncol 36(6): 589-593.

17. Hahmann M, Lueck HJ, Poelcher M, Wimberger P (2009) Surgery for recurrent ovarian cancer: role of peritoneal carcinomatosis: exploratory analysis of the desktopi trial about risk factors, surgica implications, and prognostic value of peritoneal carcinomatosis. Ann Surg Oncol 16(5): 1324-1330.

18. Sugarbaker PH (1999) Management of peritoneal-surface malignancy: the surgeon's role. Langenbeck's Archives of Surgery 364(6): 576-587.

19. Spiliotis E, Halkia E, Lianos N Cytoreductive (2015) Surgery and HIPEC in Recurrent Epithelial Ovarian Cancer: A Prospective Randomized Phase III Study Ann Surg Oncol 22(5): 1570-1575.

20. Helm CW, Richard SD, Pan J, Bartlett D (2010) Hyperthermic intraperitoneal chemotherapy in ovarian cancer: first report of the HYPER-0 registry. Int J Gynecol Cancer 20(1): 61-69.
21. Pedro Antonio, Cascales-Campos J (2014) Treatment of Microscopic Disease with Hyperthermic Intraoperative Intraperitoneal Chemotherapy After Complete Cytoreduction Improves Disease-Free Survival in Patients with Stage IIIC/IV Ovarian Cancer. Ann Surg Oncol 21(7): 2383-2389.

22. Schorge JO, Garrett LA, Goodman A (2011) Cytoreductive surgery for advanced ovarian cancer: quo vadis Oncology 25(10): 928-934.

23. Ingmar Königsrainer, Philipp Horvath (2014) Cytoreductive surgery and hyperthermic intraperitoneal chemotherapy in recurrent epithelial ovarian cancer with peritoneal metastases: a single center experience Langenbecks Arch Surg 399(5): 589-594.

24. Rene Warschkow, Ignazio (2012) Tarantino Does hyperthermic intraoperative chemotherapy lead to improved outcomes in patients with ovarian cancer? A single center cohort study in 111 consecutive patients Patient Safety in Surgery p. 6-12

25. Mulier S, Claus JP, Dierieck V (2012) Survival benefit of adding hyperthermic intraperitoneal chemotherapy (HIPEC) at the different time-points of treatment of ovarian cancer: review of evidence. Curr Pharm Des 18(25): 3793- 803.

26. Halkia E, Spiliotis J, Sugarbaker P (2012) Diagnosis and management of peritoneal metastases from ovarian cancer. Gastroenterol Res Pract pp. 541-842.

27. Aditi Bhatt, Olivier Glehen (2016) The role of Cytoreductive Surgery and Hyperthermic Intraperitoneal Chemotherapy (HIPEC) in Ovarian Cancer: A Review. Indian J Surg Oncol 7(2): 188-197.

\section{Your next submission with Juniper Publishers will reach you the below assets}

Commons Attribution 4.0 License

DOI: 10.19080/CTOIJ.2017.06.555677 